\title{
Geometric Design Rule Check of VLSI Layouts in Distributed Computing Environment
}

\author{
S.K. NANDY \\ Laboratory for Computer Aided Design, Super Computer Education and Research Center, Indian Institute of \\ Science, Bangalore, INDIA
}

(Received May 11, 1989, Revised September 18, 1990)

\begin{abstract}
In this paper we provide a distributed solution to perform Design Rule Checking (DRC) of a layout by exploiting either spatial independence or layer independence in layout data. We show that the former approach to DRC can result in reasonable speedup only for large layouts, whereas, the latter approach shows a better performance for smaller layouts. We also provide an algorithm to optimally partition a layout and a scheme to allocate DRC tasks to idle processors in a Distributed Computing Environment (DCE) to attain load balancing.
\end{abstract}

Key Words: Distributed CAD tools; Layout verification; VLSI algorithms; Distributed Computing; Custom Design tools

\section{THE LAN SOLUTION}

Local Area Networks have generally been used for connecting a number of computing modules within a limited geographical space. The potential of LANs as a communication component of a parallel processing system is being increasingly realized and much work has been done on distributed computing aspects of LANs such as intertask communication and remote call implementation [1].

Parallel processing within a LAN is attractive for two main reasons. First, it enables utilization of the spare processing capacity of the increasingly powerful workstations in the LAN by distributing the computationally intensive jobs among them. Second, it provides an inexpensive way of connecting processors together in a stand-alone multiprocessor system.

It is the first of these that interests us. It is conceivable that a CAD environment could be configured as a number of workstations connected in a LAN, with each of them being used in single-user mode as before. However, the computationally intensive tools such as a design rule checker (DRC) or a circuit extractor could be implemented as distributed algorithms. Whenever such a tool is used, it can break up the processing job into functionally inde- pendent sets of actions called sub-tasks. Some of these tasks can be scheduled on to the less computationally loaded processors on the network to be run as background tasks. After the execution of all these sub-tasks, the output results can be collected, collated and a single result presented to the user by the parent task. This of course presumes the existence of the multiprogramming capabilities provided on most of the current generation workstations that run UNIX or a similar operating system.

The approach to design such a tool would be to study the data and processing requirements of the tool and then to identify functionally independent modules. This means that the algorithms used in the design of the tool should comprise of relatively independent tasks. The exchange of data between tasks during execution should be minimized since such exchange of data would load the communication medium (the LAN) and severely restrict the realizable advantages of parallelism. Clearly on the LAN, these tasks may be scheduled on different processors if available.

In this paper we investigate the design criteria of a DRC tool in a distributed environment. Our approach is to characterize the requirements of the DRC tool [2,3] and identify the parallelism at a macroscopic level. Following this, DRC tasks are generated which are to be distributed amongst the 
available processors. Allocation of such tasks are carried out in a fashion such that all processors are maximally utilized. With regard to a CAD environment we will, for the rest of the paper, refer to CAD workstations as processors. Further, we also assume that all processors can be connected as a LAN. In a LAN, a processor will also be referred to as a node.

\section{EXPLOITING SPATIAL INDEPENDENCE IN LAYOUT DATA}

Design rule checkers fall in the category of CAD tools that operate on data with spatial dependency and this dependency is localised within a small distance specified by a design rule. We shall henceforth refer to this distance as the Design Rule Interaction Distance (DRID) [4]. It is therefore possible to partition a layout into sublayouts as shown in Fig. 1 and perform DRC as a task in parallel on these sublayouts. Such a partitioning would report false errors at the partitioned boundaries. This can be avoided if we permit the two DRC tasks on adjacent partitions (of the original layout) to communicate with each other. Such communications cause unavoidable system overheads and problems of synchronization. We overcome these by allowing overlaps between partitioned blocks of the layout by an amount equal to DRID on all sides of the partition (see Fig. 1).

\section{Mapping of the DRC problem}

The mapping of the DRC problem on to a Distributed Computing Environment (DCE) is based on exploitation of spatial independence in layout data [5]. This is achieved in two steps. The layout is first partitioned into as many sublayouts as the desired number of DRC tasks. Each sublayout, thus comprises a Task Input File (TIF) for a DRC task. DRC tasks are then allotted to the idle processors in the DCE based on an efficient task scheduling mechanism described in a later section.

\section{Layout Partitioning}

The crucial aspect of the partitioning process is to find out the optimal number of partitions. If the number of the partitions is small, then it reduces the scope of parallelism. If the number of partitions is large, then the computation time due to overlaps at the sides of the sublayouts (DRID) will be comparable to the computation time required to perform DRC on the sublayout itself (without overlaps). Further, all nodes in a DCE may not be homogeneous in terms of its computing resources. Hence, the size of a partition is limited by the available working buffer of the node with minimum computing resources. Based on this size, the number of desired partitions $\mathrm{N}$ can be determined. We then use the partitioning algo-

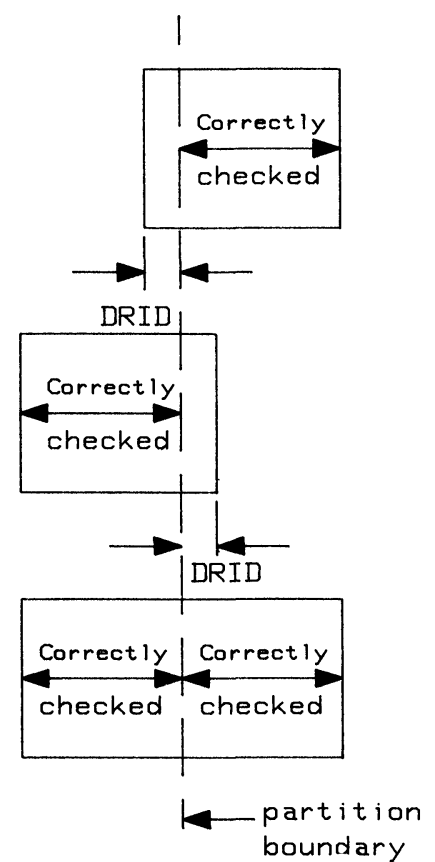

(a)

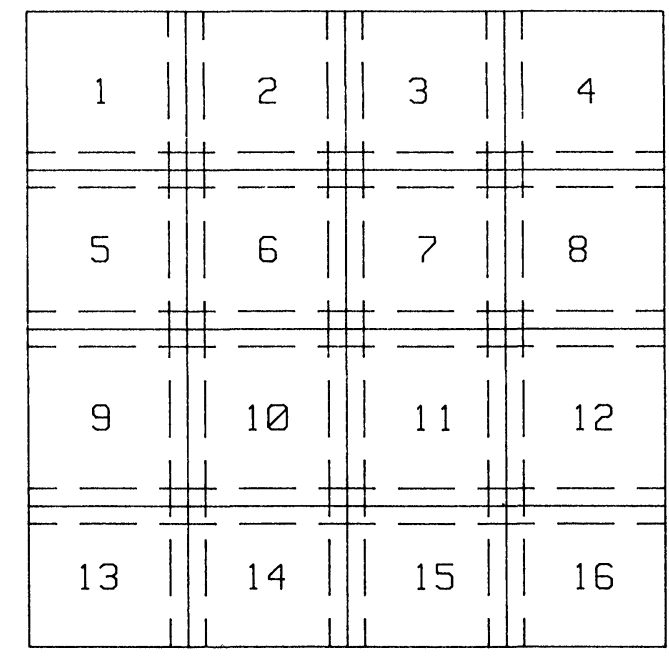

(b)

FIGURE 1 Design Rule Interaction Distance (DRID) a) Incorrectly Checked areas are within a DRID from the Partition Boundary b) Layout Partitions with DRID Overlaps. 
rithm to arrive at an optimal partition of the layout into rectilinear regions which minimizes the computation time due to overlaps of DRID along the sides of the sublayouts.

The geometric partitioning of a square VLSI layout into $\mathrm{N}$ rectilinear regions of equal areas with minimum internal wall length is a computational geometry problem [6,7]. Stated as an optimization problem, the problem aims at minimizing the internal wall length of $\mathrm{N}$ partitions of a given layout. Each partition should have the same area and no two partitions should overlap. (Note that overlap between two partitions is different from DRID overlaps.) The area constraint constitutes the set of quadratic constraints and the overlap constraint constitutes the set of linear constraints. Without loss of generality, we say that the VLSI layout is an unit square. With any region $i$ are associated coordinates $\left(a_{i}, b_{i}\right)$ and $\left(c_{i}, d_{i}\right)$ which correspond to the bottom left corner and top right corner respectively. Formally,

Minimize $\sum_{i=1}^{N} 2\left(\left(c_{i}-a_{i}\right)+\left(d_{i}-b_{i}\right)\right)-4$

subject to,

$$
\begin{aligned}
& \forall \text { region } i,\left(c_{i}-a_{i}\right)\left(d_{i}-b_{i}\right)=1 / N ; \\
& \forall \text { region } j \varepsilon T_{i} b_{j}=d_{i} ; \forall \text { region } j \varepsilon R_{i} a_{j}=c_{i} ; \\
& \forall \text { region } j \varepsilon B_{i} d_{j}=b_{i} ; \forall \text { region } j \varepsilon L_{i} c_{j}=a_{i} ; \\
& 0 \leq a_{i} \leq c_{i} \leq 1 \text { and } 0 \leq b_{i} \leq d_{i} \leq 1 ;
\end{aligned}
$$

where,

$$
\begin{gathered}
T_{i}=\{j \mid \exists \text { atleast one }(x, y) \text { in region } j \\
\text { and } \left.a_{i} \leq x \leq c_{i} \text {, and } y=d_{i}\right\} \\
R_{i}=\{j \mid \exists \text { atleast one }(x, y) \text { in region } j \\
\text { and } \left.x=c_{i} \text {, and } b_{i} \leq y \leq d_{i}\right\} \\
B_{i}=\{j \mid \exists \text { atleast one }(x, y) \text { in region } j \\
\text { and } \left.a_{i} \leq x \leq c_{i} \text {, and } y=b_{i}\right\} \\
L_{i}=\{j \mid \exists \text { atleast one }(x, y) \text { in region } j \\
\text { and } \left.x=a_{i} \text {, and } b_{i} \leq y \leq d_{i}\right)
\end{gathered}
$$

The general optimization problem with a linear objective function and quadratic constraints has been proved to be NP-Hard in $[8,9]$. We are interested in sub-optimal heuristic algorithms with good per- formance bounds. We give a sub-optimal linear time heuristic for solving this problem.

\section{Partitioning Algorithm}

Without loss of generality we assume the VLSI layout to be an unit square. If the number of desired partitions $N$ is a perfect square, then the unit square is partitioned into $N$ squares each of area $1 / N$. If $N$ is not a perfect square, then we can divide the layout into $N$ equal partitions (of area $1 / N$ ) using one of the two schemes given below.

Scheme 1: In this scheme, we divide the entire layout into floor $(\sqrt{ } N)$ columns. All columns except the last, are partitioned into ceiling $(\sqrt{ } N)$ rows. The last column accommodates the remaining number of partitions.

Scheme 2: The layout is divided into ceiling $(\sqrt{ } N)$ columns. All columns except the last, are divided into floor $(\sqrt{ } N)$ rows. The last column is partitioned into the remaining number.

The partitioning algorithm $\operatorname{Partition}(N)$ is presented below in Pseudo Pascal:

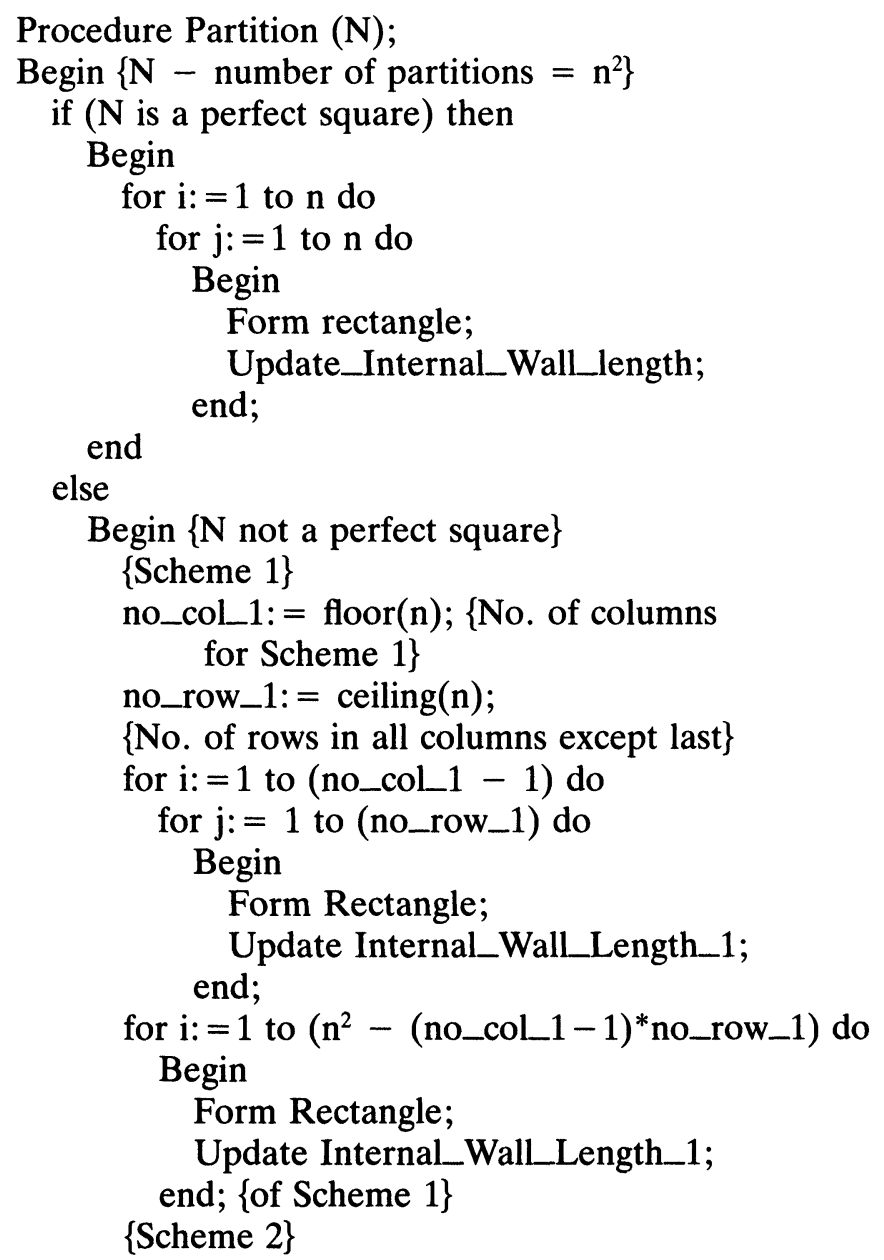




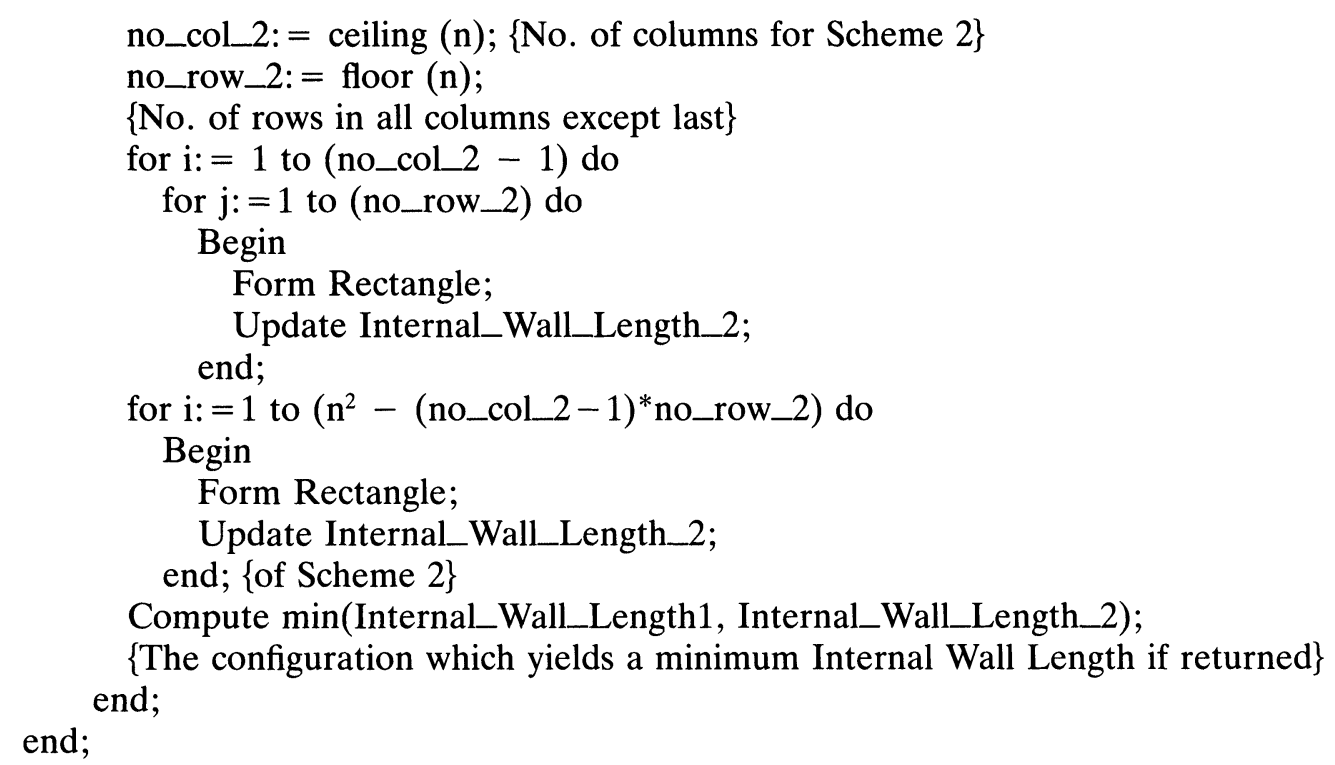

\section{EXPLOITING LAYER INDEPENDENCE IN LAYOUT DATA}

Design rules are constraints imposed on the extent of a mask opening corresponding to individual layout geometries (LGs) in a layout. Within a layout, width of design rules vary for different layers. Also, there exist layers where there are design rule interactions among them (such as poly and diffusion), and there are layers (such as poly and metal) that may not have any design rule interactions [10]. Parallelism in DRC can thus be exploited by partitioning the layout into sets of noninteracting layers, and performing DRC on them. A DRC task will thus operate on layout data pertaining to the set of interacting layers. Clearly, using such a scheme, the maximum number of DRC tasks is limited to a maximum of the number of layers associated with a layout. The advantage of this scheme is layout partitioning at no extra cost. The disadvantage of this scheme is increased memory requirements, since data of interacting layers must be replicated in the processors.

\section{DISTRIBUTED TASK ALLOCATION}

In this section we provide a task allocation algorithm which is distributed (i.e. the algorithm runs in all the nodes of the distributed computer system). The algorithm is general, and is not restricted to DRC tasks alone. It can be adopted by a class of distributed physical VLSI design tools, which spawns CAD tasks corresponding to every partition of the layout to speed up the design.
The proposed task allocation algorithm is based on the assumption that each node in the DCE has a means of measuring its state. The state signifies the number of tasks that are currently queued and serviced in the node. The algorithm is based on a Threshold Policy $[11,12]$. The node's status information dictates whether the node is an Idle node, a Neutral node or a Busy node. An Idle node is one which can accept tasks from the other nodes of the DCE. A Busy node is one which can give tasks to the other nodes of the DCE. A Neutral node is one which can neither accept tasks nor give tasks. In other words we strive to make all nodes neutral to balance the load. We define a vector called the load vector whose elements specify the status of the various nodes in the DCE at any instant. We define another vector $B$ called the belonging vector, which is maintained in every node of the DCE. It is used for computing Interprocess Communication (IPC) costs. $B=\left(b_{1}, b_{2}, \ldots, b_{k}\right)$ where $k$ is the total number of DRC tasks in the system. Each element $b_{i}$ is defined as follows:

$$
\begin{aligned}
b_{i} & =1 \text { if task } i \text { is present at the node in question } \\
& =0 \text { otherwise. }
\end{aligned}
$$

There are primarily two ways to detect a significant change in the load of a node. One way is to allow the loaded or Busy node in the system to query its neighbors to accept tasks in order to decrease its own load. The second way is to make an Idle node request tasks from its neighbors. We adopt the latter policy since it is better to execute the tasks that are already 
present in a Busy node than wasting time in querying and waiting for some node to accept tasks from it. On the other hand, when the system on the whole is lightly loaded, the number of queries from Idle nodes and hence the processing cost for queries is high. This however is partly offset by the fact that most of the nodes are Idle. Therefore, whenever a node becomes idle it queries its neighbors requesting their status. The rate at which an idle node queries its neighbors is determined by a factor called PERIODICITY. Each node maintains a window, and any node falling within the window of this node is a neighbor node. The window of size $w$ is defined by selecting $w / 2$ nodes on either side (of the node in the load vector). The concept of a window leads us to a multicast policy. The case where the window size is equal to the number of nodes in the DCE corresponds to a broadcast. The advantages of multicast over broadcast has been discussed in [13]. Note that a node can occur in more than one window. When a node receives the status requested message it may do one of the following based on the node's state: If the queried node is a busy node, it sends its status to the querying node. If it is a neutral or an idle node, the queried node does not respond at all. The querying node will timeout if no status is received within a predetermined time. Once the querying node receives responses to the query it stores the address and status of the responding nodes in a vector called the task allocation vector and invokes the Assign_Task procedure. The number of tasks that can be accepted by this node (say, $x$ ) is computed by the Task_Allocation procedure using the Threshold.

We define the number of ripples to be the number of times the idle nodes in the DCE embroil themselves in accepting tasks. In other words it is the total number of the Assign_Task procedure invocations for the DCE as a whole.

The Assign_Task procedure computes the number of tasks that can be offloaded from the busy nodes to the idle node, and updates the allocation vector in one of the following ways:

1. The allocation vector is first sorted in descending order of the status key. The ceiling of the quotient of the number of tasks that can be accepted divided by the size of the allocation vector, will give the number of tasks to be fetched from one element of the vector in one iteration. The iterations are continued till there are no more tasks that can be accepted or no more tasks that can be supplied. The latter case occurs when the sum of all the elements of the allocation vector is less than the number of tasks that can be accepted by this node.
2. The selection of an element from the vector is random. Once an element is selected it is compared with $x$. If it is greater than $x$, then the requirement has been met and a request for $x$ number of tasks can be sent to the corresponding Busy node. Otherwise, the probability of choosing the already chosen element is made zero, and another element from the allocation vector is chosen randomly and the same process is repeated.

The reason behind using a random order assignment in the Assign_Task procedure is as follows. For instance, consider a load vector $<18,10,4,16,14,4,16,20>$ corresponding to the nodes $n_{1}, n_{2}, n_{3}, n_{4}, n_{5}, n_{6}, n_{7}$ and $n_{8}$ and a window size of 4 with a Threshold of 6 . Since we allow window overlaps, by descending order allocation, the not so heavily loaded nodes (eg. $n_{5}$ ) are deprived of offloading their tasks to the Idle nodes. This is because priority is given to the most heavily loaded nodes. By random order allocation, there is a scope for the not so heavily nodes to offload. Further, the descending order allocation policy does not contribute to the global load balancing. The random order allocation policy avoids a local minima although the basic algorithm is inherently greedy.

Once the above process is complete the Idle node sends the task requested query with the appropriate number of tasks requested and the belonging vector to the Busy node identified by the Assign_Task procedure. After transmitting the tasks requested query, the idle node does a timeout and receives the tasks sent by its busy neighbors.

When a busy node receives the tasks requested query from one of its idle neighbors the busy node first computes the advantage a DRC task can gain by migrating the task from it to the idle node. This is done by the procedure Estimate_Advantage. The Estimate_Advantage procedure first takes a DRC task from the rear of the job queue and finds out the tasks with which it has to communicate (IPC). The hamming_distance procedure finds out whether two DRC tasks correspond to two adjacent layout partitions. Further, in the Estimate_Advantage procedure, the increase in IPC cost for the particular DRC task when migrated to the idle node as well as the IPC costs which the node would be saving after migrating the task to the idle node is computed with the aid of the busy node's belonging vector and the idle node's belonging vector. A net IPC cost that has to be incurred is also computed. A weighting factor for different processor speeds in the case of an heterogenous DCE, can also be introduced here. If the queueing delay in this node is greater than the sum of queueing delay in the idle node and the weighted 
IPC cost, then there is gain by migrating that DRC task. Hence the task is migrated.

A Pseudo Pascal description of the Task_Allocation algorithm is given in the appendix.

\section{ALGORITHM FOR DISTRIBUTED DRC}

A node which acts as a host for the entire run of DRC obtains the number of idle processors in the DCE and partitions the layout data into appropriate number of Task Input Files (TIF). The TIFs are then allocated by the host to the idle nodes in the DCE. Following this, the host sends a control packet to all the participating nodes. This control packet contains appropriate commands to run a DRC task and to return error files to the host. The description of the algorithm for distributed DRC is given below using structured English.

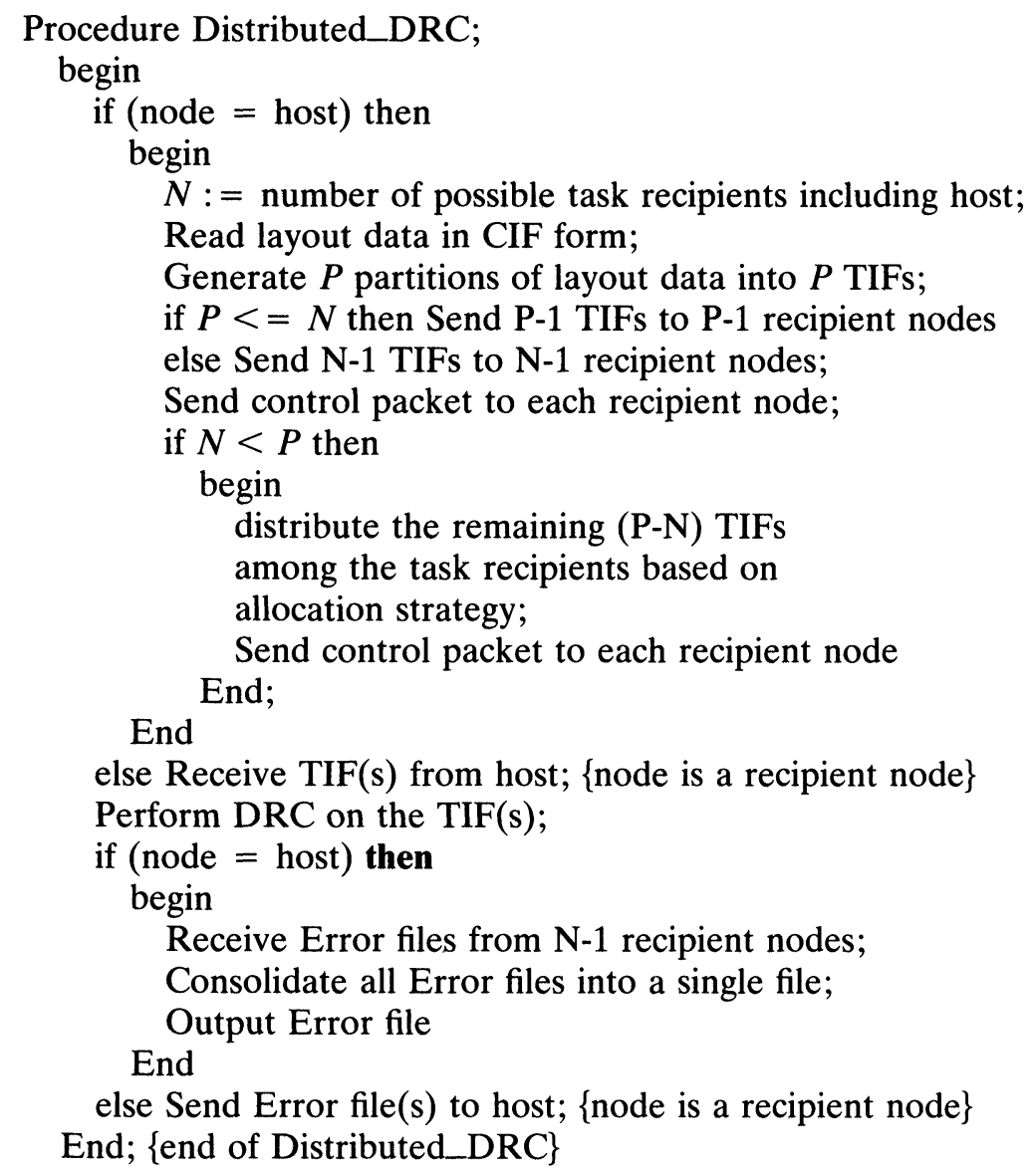

ated on the NELSIS IC Design System (VLSI CAD Tools from the Delft University, The Netherlands), using the custom layout tool DALI. All circuits are flat. DALI generates $<$ files.ldm $>$, that stores information about the LGs in a format similar to CIF. All experiments were conducted on the APOLLO DN 3000 with MC68020 as the CPU (12MHz Clock) with 68881 numeric coprocessor. The distributed DRC was tested on three machines connected in a Ring. The partitioning scheme adopted is based on that which exploits spatial independence in layout data to generate the TIFs. All TIFs were statically allocated by the host to other nodes in the DCE. The optimum allocation of TIFs on the idle nodes in the DCE was obtained through a simulated execution of the distributed task allocation algorithm. (Performance of the distributed task allocation algorithm is presented in the following section.) Using the

\section{SIMULATION RESULTS}

We present the execution time for design rule check of different circuits below. These circuits were cre- files.ldm in a TIF, linear quadtrees corresponding to each layer were created. The layout as stored in quadtrees, were the inputs to the DRC algorithm. The algorithm for DRC is based on a linear quadtree 
representation of mask layouts $[14,3,2]$ and has a linear time performance with respect to the number of layout geometries in the layout. The implemented version of DRC does not cater to context sensitive checks. Since all experiments were carried out on three identical workstations connected in a ring, there was no reallocation of tasks during execution. Table 1 summarizes the results. The cost of generating layout partitions is not included in the results because they were carried out separately on files.ldm and has negligible contribution compared to the cost of $\mathrm{I} / \mathrm{O}$ and file transfers which are included in the results in Table 1.

From the values in Table 1, it is evident that the use of three machines results in reasonable speedup only for large layouts. This is as expected since for smaller layouts the cost of network file transfer and $\mathrm{I} / \mathrm{O}$ nullifies the gain due to parallelism. Yet another reason for the low speedup in smaller layouts is due to the fact that the LGs are not uniformly distributed. This results in some partitions having more LGs than the other partitions. Nevertheless, in real life VLSI Design we can expect higher speedups since in real VLSI layouts, LGs are uniformly distributed and network file transfer cost will be negligible when compared to the cost of computation. For a realistic estimate consider a layout partition of 1024 lambda $\times$ 1024 lambda. If 1 lambda $=1 \mu$, then this layout partition corresponds to a layout area of $1 \mathrm{~mm} \times$ $1 \mathrm{~mm}$ with approximately $10,000 \mathrm{LGs}$, where each LG corresponds to approximately 3 CIF rectangles. Using our algorithm, time required to perform DRC on such a layout partition is approximately $5420 \mathrm{sec}-$ onds. Since a TIF contains information about LGs that are stored in a format identical to that in files.ldm (i.e. layer number and coordinates of all vertices in a LG), it is realistic to assume a storage of 50 bytes/ LG (corresponding to its layer number and 12 corners) on the average. Thus, the size of a TIF is approximately 500 Kbytes. If we consider the time for reallocation of tasks in a DCE to be equal to the time required to transfer a TIF from one node to another, a typical estimate of the cost of file transfer

TABLE 1

Results of DRC in a distributed computing environment

\begin{tabular}{|c|c|c|c|c|c|c|c|}
\hline \multirow[b]{2}{*}{ Circuit } & \multicolumn{4}{|c|}{$\begin{array}{l}\text { No. of boxes } \\
\text { in partition }\end{array}$} & \multicolumn{2}{|c|}{ Time (in secs) } & \multirow[b]{2}{*}{ Speedup } \\
\hline & 1 & 2 & 3 & Orig & Sequential & Parallel & \\
\hline Inverter & 38 & 12 & 0 & 50 & 100.84 & 90.2 & 1.12 \\
\hline Latch1 & 64 & 48 & 0 & 104 & 114.57 & 87.62 & 1.3 \\
\hline Latch2 & 60 & 52 & 40 & 133 & 123.18 & 105.4 & 1.17 \\
\hline Adder & 233 & 111 & 193 & 491 & 170.1 & 130.15 & 1.31 \\
\hline Counter & 274 & 128 & 205 & 592 & 187.1 & 134.53 & 1.4 \\
\hline
\end{tabular}

TABLE 2

Performance of DRC in a DCE on exploiting layer independence between DRC tasks

\begin{tabular}{lccc}
\hline & \multicolumn{2}{c}{ Time (in secs) } & \\
\cline { 2 - 3 } Circuit & Sequential & Parallel & Speedup \\
\hline Inverter & 92.12 & 33.24 & 2.77 \\
Latch1 & 97.46 & 43.58 & 2.23 \\
Latch2 & 105.74 & 48.44 & 2.18 \\
Adder & 134.70 & 72.42 & 1.84 \\
Counter & 148.52 & 77.54 & 1.93 \\
\hline
\end{tabular}

is 25 seconds with a sustained data transfer rate of $20 \mathrm{Kbytes} / \mathrm{sec}$ in the LAN. This contributes to an overhead which is within $1 \%$ of the execution time of DRC. It is thus reasonable to conclude that the distributed DRC algorithm will scale with an increasing problem size.

We also carry out simulation studies to evaluate the performance of parallel DRC that exploits layer independence in layout data for generating TIFs. Due to limitations of disk space, the above parallel algorithm was simulated on the HP 9000/320 system, in an HP-UX environment. Three processors were simulated spawning three DRC tasks using the UNIX fork() call. Communication between processes is accomplished using pipes. In the simulation results provided in Table 2, we have assumed the existence of a file server. This permits us to hide the actual cost of file transfer since only the file pointers to the respective TIFs are sent by the host to the recipient nodes in the DCE.

It can be observed from the Table 2, that there is a fall in speedup as the size of the circuit increases. Reasons for such a behavior can be attributed to the following. Each TIF (layout partition) is associated with a set of interacting layers. The nature and the extent of interaction depends on the design rule that is being checked. Consequently, the difference in computation requirements of individual TIFs is more pronounced as the size of the circuit increases. Since speedup is determined by the process that executes for the longest duration, speedup falls with the increase in circuit size. This behavior is in contrast to the case when the partitioning scheme exploits spatial independence of layout data. However, the speedup figures in Table 1 cannot be compared to that in Table 2 since all execution times in Table 1 are by the time of the clock and include the cost of file transfer and $\mathrm{I} / \mathrm{O}$. Instead, if we assume the existence of a server [15] in each node of the DCE (which exploits spatial independence in layout data), the speedup figures in Table 1 appear as shown in Table 3.

From Table 3, it can be concluded that distributed execution of DRC tasks using a partitioning scheme 
TABLE 3

Performance of DRC in a DCE with a server on exploiting spatial independence

\begin{tabular}{lc}
\hline Circuit & Speedup \\
\hline Inverter & 1.16 \\
Latch1 & 1.34 \\
Latch2 & 1.25 \\
Adder & 1.67 \\
Counter & 2.05 \\
\hline
\end{tabular}

that exploits spatial independence performs better than the one that exploits layer independence in layout data for large circuits. We can thus expect higher speedup in DRC for layouts of VLSI circuits and regular layouts.

\section{ANALYSIS OF THE PARTITIONING AND TASK ALLOCATION ALGORITHMS}

Our approach to task partitioning is to partition the VLSI layout into $N$ equal areas with the condition that overlaps of DRID along the sides of each partition and interaction between processors are minimal. Note that each partition of the VLSI layout constitutes data for a separate DRC task. We also require that each partition is rectilinear. It turns out that minimizing overheads is the same as minimizing the internal edge lengths of all the partition boundaries that are internal to the original layout. When $N$ is a perfect square there are $\sqrt{ } N$ rows and $\sqrt{ } N$ columns. Forming a rectilinear region can be done in constant time since all we need to calculate is the length and breadth of the rectilinear region. Hence it takes $\sqrt{ } N^{*} \sqrt{ } N$ time to partition the given layout into $N$ rectilinear regions. When $N$ is not a perfect square, partitioning using either Scheme 1 or Scheme 2 is also bound by $[\sqrt{ } N]^{*}[\sqrt{ } N \mid$ which is $0(N)$. Thus we have $0(N)$ heuristic for solving the problem.

\section{Lemma 7.1}

The perimeter of a rectilinear region of a fixed area $\mathrm{A}$, is minimum when the length and breadth of the rectilinear region are equal.

\section{Proof}

Obvious. Hence the lemma is proved.

\section{Theorem 7.1}

If $N$ is a perfect square, the partitioning Algorithm yields an optimal solution.

\section{Proof}

The problem requires $N$ partitions of equal areas. The partitioning algorithm yields $N$ partitions where each partition is a square. The problem of minimizing the internal wall lengths is the same as minimizing the perimeters of all the $N$ rectilinear regions. Since a square will yield the minimum perimeter for a given area (from Lemma 7.1), the sum of the perimeters of all the $N$ squares will be the minimum possible wall length for any configuration of $N$ partitions. Thus the partitioning algorithm gives the optimal solution. Hence, the theorem is proved.

In the following, we give performance guarantees when $N$ is not a perfect square. Further we determine $\gamma$, the lower bound on the optimal value of Internal Wall Length. Without loss of generality, we assume that the VLSI layout is an unit square.

\section{Lemma 7.2}

The minimum possible Internal Wall Length for any $N$, with overlaps of any two rectilinear regions allowed, is given by $4(\sqrt{ } N-1)$.

\section{Proof}

From Lemma 7.1 we see that the square partition will have the minimum wall Length. If we remove the constraint that no two rectilinear regions can overlap, the unit square is a partial covering for the $N$ squares each of side $1 / \sqrt{ } N$. The total Internal Wall Length, IWL is given by,

$$
\mathrm{IWL}=2 N(1 / \sqrt{ } N+1 / \sqrt{ } N)-4=4(\sqrt{ } N-1)
$$

It follows that the above value of internal wall length corresponds to an optimal partition of $N$ from Theorem 7.1. Hence the lemma is proved.

From lemma 7.2 it is obvious that IWL is also a lower bound on the optimal solution of the partitioning problem for any $N$ with the constraint that no two rectilinear regions can overlap. Hence $\gamma=$ IWL.

We now prove that we obtain a near optimal solution asymptotically using the two heuristic partitioning schemes proposed earlier in the section Partitioning Algorithm. To establish the near optimality of the partitioning schemes, it is sufficient to compare the internal wall length obtained through the partitioning schemes with $\gamma$, since $\gamma$ is a lower bound on the optimal solution. Let IWLH1 and IWLH2 represent the internal wall lengths obtained using Scheme 1 and Scheme 2 respectively. We consider partitioning of a unit square area into $N\left(=n^{2}\right)$ partitions.

\section{Theorem 7.2}

Partitioning a layout using either Scheme 1 or Scheme 2 yields a near optimal internal wall length asymptotically. 


\section{Proof}

For Scheme 1, we note the following:

No. of columns $=\lfloor n\rfloor$

No. of rows in every column except last $=[n]$

No. of rows in last column $=n^{2}-(|n|-1)^{*}[n]$

$$
=n^{2}-[n]^{*}[n]+[n]
$$

IWLH1 $=$ Perimeter of all partitions -4

IWLH1 $+4=2 *(\mid n\rfloor-1) *[n] *(1 /[n]+[n] /$

$\left.n^{2}\right)$

$+2 *\left(n^{2}-\lfloor n] *[n]+[n]\right) *(1 /$

$\left(n^{2}-\lfloor n\rfloor *[n]+\lceil n]\right)$

$\left.+\left(n^{2}-[n] *[n]+[n]\right) / n^{2}\right)$

Simplifying we obtain

$(\mathrm{IWLH} 1+4) / 2=\underset{n^{2}}{\lfloor n\rfloor}-(\lceil n\rceil *\lceil n\rceil *\lfloor n\rfloor) / n^{2}+$

$+(\lfloor n\rfloor *\lfloor n\rfloor *[n\rceil *[n]) / n^{2}-$

$(2 *\lfloor n\rfloor *[n])+2 *[n]$

Substituting $[n]=|n|+1$ and simplifying, we have

$$
\begin{aligned}
(\mathrm{IWLH} 1+4) / 2= & {[n\rfloor^{4} / n^{2}+\lfloor n\rfloor^{3} / n^{2}+n^{2}+\lfloor n\rfloor } \\
& +2 \\
& -\left(2 *\lfloor n\rfloor^{2}+\lfloor n\rfloor / n^{2}+\lfloor n\rfloor^{2} /\right. \\
& \left.n^{2}\right)
\end{aligned}
$$

Substituting the upper bound for $\lfloor n\rfloor$ as $n$, we obtain

R. H. S. $=2^{*} n+1-1 / n$

Substituting the lower bound for $[n]$ as $n-1$, we obtain

R. H. S. $=2 * n+1$

Therefore,

$($ IWLH1 +4$) / 2 \leq 2^{*} n+1$ and IWLH1

$$
\leq 4^{*} n-2
$$

i.e., IWLH $1 \leq 4 * \sqrt{ } N-2$

Since $\gamma=4^{*} \sqrt{ } N-4$,

$$
\frac{\text { IWLH1 }}{\gamma} \leq \frac{4^{*} \sqrt{ } N-2}{4^{*} \sqrt{ } N-4}
$$

Similarly, it can be shown that

$$
\frac{\mathrm{IWLH} 2}{\gamma} \leq \frac{4^{*} \sqrt{ } N-2}{4^{*} \sqrt{ } N-4}
$$

Thus the partitioning algorithm gives near optimal partition of a layout asymptotically.

The Distributed Task Allocation algorithm presented earlier, strives to attain load balancing in the DCE. The crucial step in the Task_Allocation procedure is the Assign_task step wherein a descending order schedule is followed to arrive at the number of tasks to be requested by the idle node to the busy nodes in the window. This consists of a sorting phase where the neighbors in the idle node's window (a maximum of $x$ ) sorted with their status as the key. Besides the sorting phase, the number of invokations $y$ of the Assign_Task is bound by the number of tasks that can be accepted by the idle node. Hence the algorithm's time complexity is $0(x \log x+y)$ where $(x \log x)$ is the contribution of the sorting phase.

A DCE was emulated on the APOLLO DN3000 workstations, in programming language $\mathrm{C}$ under UNIX BSD4.2 environment. Each processor is simulated by a process which was spawned by the UNIX fork () call. Communication between processes is accomplished by means of pipes. The code is written in such a manner that studies can be done for topologies like Ring, Bus and Mesh.

For the purpose of quantifying Load Balancing we introduce a measure known as the UNBALANCE FACTOR. Since ours is a threshold policy, we assign numbers for each range of the status. We fix the threshold of MEDMIN at 6 and that of MEDMAX at 10 . A node is assigned the number 1 if $s$, the status of the node is $1 \leq s<4,2$ if $4 \leq s<$ MEDMIN, 3 if MEDMIN $\leq s<$ MEDMAX, 4 if MEDMAX $\leq$ $s<15,5$ if $15 \leq s<22$. For all other values of status, a node is assigned the number 6 . We define the UNBALANCE FACTOR as follows:

UNBALANCE FACTOR $=\left(\Sigma\left(n_{i}-\mathrm{NBAR}\right)^{2}\right) / N$

where $n_{i}$ is the number assigned to the ith node and NBAR is the number assigned to a Neutral node; a Neutral node is the one whose status lies between MEDMIN and MEDMAX. Hence NBAR is assigned 3.

Our objective then would be to bring down the Unbalance Factor to zero. To verify how close our algorithm achieves this we conducted the test of finding the variation of the UNBALANCE FACTOR for a particular load vector with respect to various window sizes. In all the simulation runs, the parameters were set to the following values:

MEAN $=8$, MEDMIN $=6, \operatorname{MEDMAX}=10$, Acceptance $=0.5$,

Expected Computation time of a task $=120 \mathrm{msec}$, Data transfer rate $=10 \mathrm{Mbits} / \mathrm{sec}$,

Packet size $=1250$ bytes,

PERIODICITY $=10 \mathrm{sec}$.

The performance of the task allocation algorithm was studied for different sizes of DCE ranging from 5 to 20 nodes. In all our studies with realistic load vectors it was observed that in order to obtain an unbalance factor close to 0 , it is of no consequence if we increase the window size beyond a certain limit, which in the case of a 5 node DCE is 4 and for a 20 
node DCE is 8 , and the corresponding number of iterations (ripples) to achieve this being 4 and 6 respectively. Further, with a $20 \mathrm{Kbytes} / \mathrm{sec}$ sustained data transfer rate in the LAN, the overhead associated with each ripple is 0.05 seconds (corresponding to an information transfer of 1 Kbyte on the LAN). Consequently, the overheads of arriving at an optimal task allocation is negligible compared to the time required for executing a DRC task. Therefore, if we keep this limit as our window size, we will attain the best possible load balancing and maintain minimum communication overheads for this unbalance factor as well. However the window size will vary for different load vectors. One way of fixing the windowsize is by studying the past history of the load conditions.

\section{CONCLUSIONS}

In this paper a distributed DRC algorithm was developed to exploit parallelism in DRC by exploiting geometric locality in layout geometries. In a distributed computing environment (DCE), we have shown that DRC can be performed simultaneously on different partitions of a layout. An instance of DRC, together with a layout partition (called DRC task), is executed in a DCE by utilizing the idle CPU cycles of workstations connected in an existing LAN. The novelty of such an algorithm is that linear speedup in DRC is obtained at no extra cost. It was shown that DRC can be performed in parallel by exploiting either spatial independence or layer independence in layout data. We also observed that the former approach to DRC can result in reasonable speedup only for large layouts, whereas, the latter approach shows a better performance for smaller layouts. We proposed an algorithm to optimally partition a layout and a scheme to allocate DRC tasks to idle processors in a DCE that strives to attain load balancing.

However, a desirable feature of any distributed CAD tool should be aimed at relieving the designer of the details of partitioning a layout and the subsequent allocation of CAD tasks (for which each layout partition constitutes a task input file) to processors in the DCE. Thus, the effort of a designer (as a user) can be limited to that of specifying the CAD Tool to be used with the data and a system call to invoke distributed execution of the CAD tool. Such a feature needs to be explored as an extension of this work.

\section{Acknowledgments}

The author gratefully acknowledges the contribution of V. Rajaraman, Chairman, Laboratory for CAD, and Supercomputer Education and Research Center, Indian Institute of Science for initiating this research and for his valuable guidance and support. The many fruitful discussions with Rajat Moona and A. Murali in arriving at the algorithms for layout partitioning and distributed task allocation is acknowledged.

\section{References}

[1] S.Y. Levy, "Distributed Computation for design aids," Proc. of the 19th ACM/IEEE Design Automation Conference, 1982.

[2] S.K. Nandy and I.V. Ramakrishnan, "Dual Quadtree representation for VLSI Designs," Proc. of the 23rd ACM/ IEEE Design Automation Conference, 1986.

[3] S.K. Nandy and L.M. Patnaik, "Linear Time Geometrical Design Rule Checker based on Quadtree representation of VLSI Mask Layouts," Computer-Aided Design, Butterworth \& Co. (Publishers) Ltd., Vol. 18, No. 7, pp. 380388, September 1986.

[4] G.E. Bier and A.R. Pleszkun, "An algorithm for Design Rule Checking on a Multiprocessor," Proc. of the 22nd ACM/IEEE Design Automation Conference, 1985.

[5] J.D. Marantz, "Exploiting Parallelism in VLSI CAD," Proc. of the IEEE International Conference on Computer Design, 1986.

[6] S. Kundu, "The equivalence of the subregrion representation and the Wall representation for a certain class of rectangular dissections", Communications of the ACM, Vol. 31, No. 6, pp. 752-763, June 1988.

[7] F.P. Preparata and M.I. Shamos, Computational Geometry, An introduction, Springer-Verlag, 1985.

[8] M.R. Garey and D.S. Johnson, Computers and Intractability: A Guide to the Theory of NP-Completeness. New York: Freeman, 1979.

[9] S. Sahni, "Computationally Related Problems," SIAM Journal of Computing, Vol. 3, No. 4, pp. 262-279, December 1974

[10] J.K. Ousterhout et al., "A collection of papers on Magic," UCB/CSD 83/154, December 1983.

[11] E. Shamir and E. Upfal, "A Probabilistic Approach to the Load Sharing Problem in Distributed Systems," Journal of Parallel and Distributed Computing, No. 4, pp. 521-530, 1987.

[12] Frank C.H. Lin and R.M. Keller, "Gradient Model: A Demand-Driven Load Balancing Scheme," Proc. of the 6th International Conference on Distributed Computing, 1986.

[13] M. Stumm, "Strategies for Decentralized Resource Management," SIGCOMM '87 Workshop, Computer Communications Review, Vol. 17, No. 5, pp. 245-252, 1988.

[14] S.K. Nandy, "Algorithms for Geometric Design Rule Checking of VLSI Layouts in Distributed Architectures," $\mathrm{Ph}$. D. dissertation, Department of Computer Science and Automation, Indian Institute of Science, 1989.

[15] Ralph-Michael Kling and P. Banerjee, "Concurrent ESP: A Placement Algorithm for Execution on Distributed Processors," Proc. of the IEEE International Conference on Computer-Aided Design, 1987.

\section{Biography}

S.K. NANDY obtained his B.Sc (Hons) Physics degree from Indian Institute of Technology Kharagpur in 1977, and subsequently obtained B.Engg. (Hons) in Electronics and Communications Engineering in 1980, MSc. (Engg.) in Computer Science and Engineering in 1987, Doctor of Philosophy in Computer Science and Engineering in 1989 from the Indian Institute of Science, Bangalore, INDIA. His research interests are in the areas of VLSI CAD, Parallel and Distributed Architectures, Dataflow Computing, Architectural Synthesis of Low Latency, High Throughput systems on silicon, High Level Synthesis, etc. He has several research papers presented at International Conferences and Journals. He is presently an Assistant Professor at the Computer Aided Design Laboratory and the Department of Electrical Communications Engineering of the Indian Institute of Science, Bangalore, INDIA. 


\section{APPENDIX}

Procedure Task_Allocation;

Begin

\{MEAN-the average number of tasks that is to be present in each node under load balanced conditions (Neutral).

MEDMIN-the threshold below which a neutral node becomes an idle node.

MEDMAX - the threshold above which a neutral node becomes a busy node.

status-the number of tasks\}

for all nodes in the DCE do

Begin

while (true) do

Begin

if (status $<$ MEDMIN) then mode $:=$ IDLE

else

if (status $>$ MEDMAX) then mode $:=$ BUSY

else mode $:=$ NEUTRAL;

case mode of

IDLE:

Begin

Request_Status;

Assign_Task;

Get_Task;

end;

BUSY:

Begin

if (Status_Requested) then

Send_Status;

if (Tasks_Requested) then

while (more_tasks_requested) do

Begin

Estimate_Advantage;

Transfer_Task;

end; end;

NEUTRAL:;

end; $\{$ case $\}$

end;

end; $\{$ while $\}$

end; \{main $\}$

Procedure Request_Status;

Begin

Send Status query to all neighbors in the window;

\{a multicast is done

Timeout for receiving status;

Form the allocation vector $\left(a_{1}, \ldots, a_{i}, \ldots, a_{n}\right)$;

\{each element $a_{i}$ of the allocation_vector will contain the number of tasks that can be given by that busy node\}

end;

Procedure Assign_Task;

Begin

\{ACCEPTANCE-decision factor for random or descending order assigning. space - the number of tasks this node can accept.

$\mathrm{n} \quad-$ the size of the allocation vector.

allocation_vector-the result of the this procedure stored here-used while requesting tasks\} 


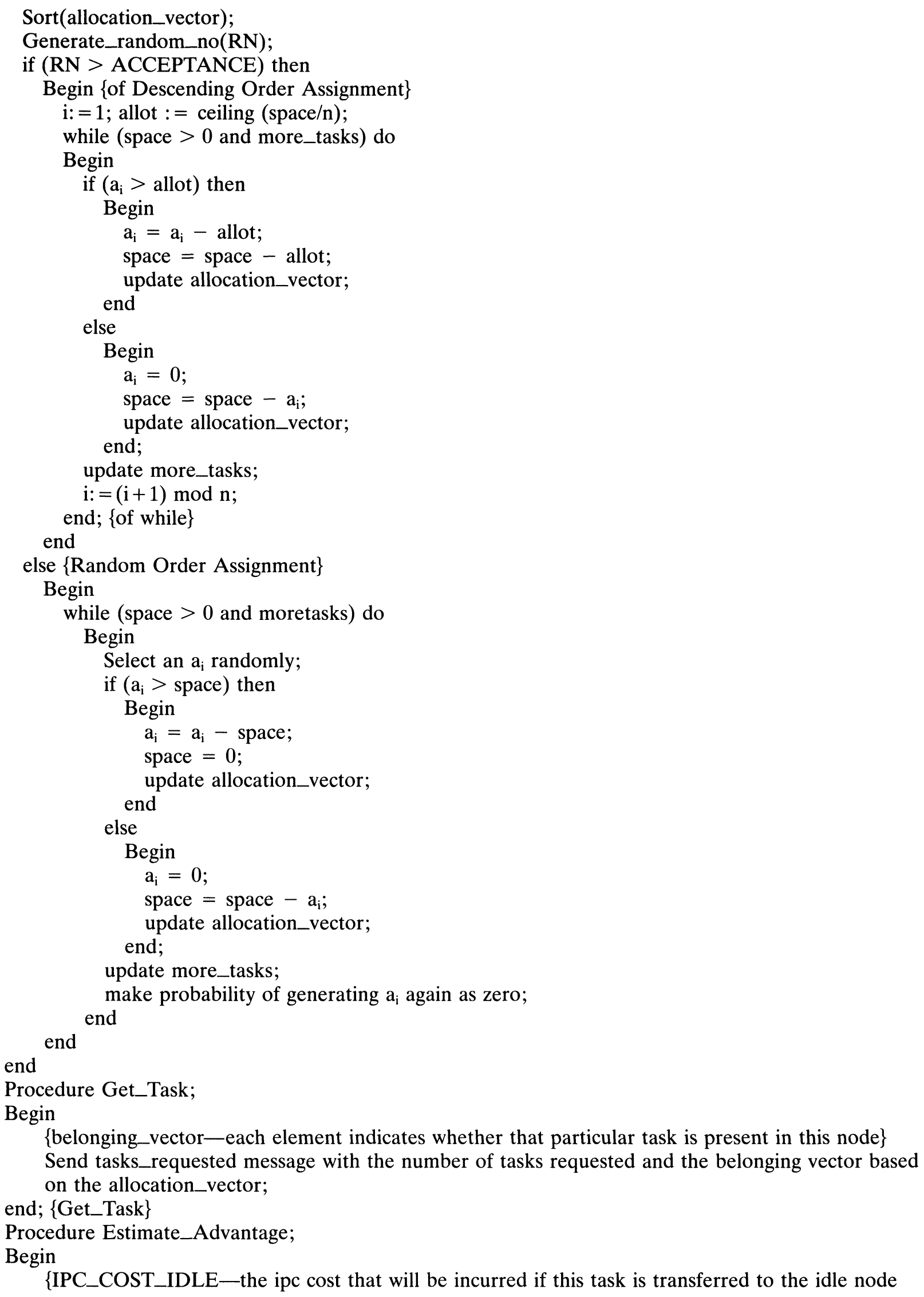


IPC_COST_HERE-the ipc cost that will be saved because of this task's transfer status_idle-status of the idle node

transfer_vector-this vector stores information about the tasks to be transferred\}

Scan the job queue from rear to take the first DRC task i; evaluate_ipc_cost (i);

if (IPC_COST_IDLE + status_idle < status + IPC_COST_HERE) update transfer_vector;

end; $\{$ Estimate_Advantage)

Procedure Transfer_Task;

Begin

Transfer tasks with their environments to the idle node based on the transfer_vector; end; $\{$ Transfer_Task $\}$

Procedure evaluate_ipc_cost(i);

Begin

for $\mathrm{j}:=0$ to size of (belonging_vector)-1 do

Begin

if (belonging_vector[j] and hamming_distance $(i, j))$ then update IPC_COST_IDLE;

if (belonging_vector[j] of idle node and hamming_distance(i,j)) then end; update IPC_COST_HERE;

end; $\{$ evaluate_ipc_cost $\}$

Procedure hamming_distance(i,j);

Begin

\{Scan neighbor list maintained in the partition\}

if ( $i$ and $j$ are neighbors) then return(1);

end; \{hamming_distance $\}$ 

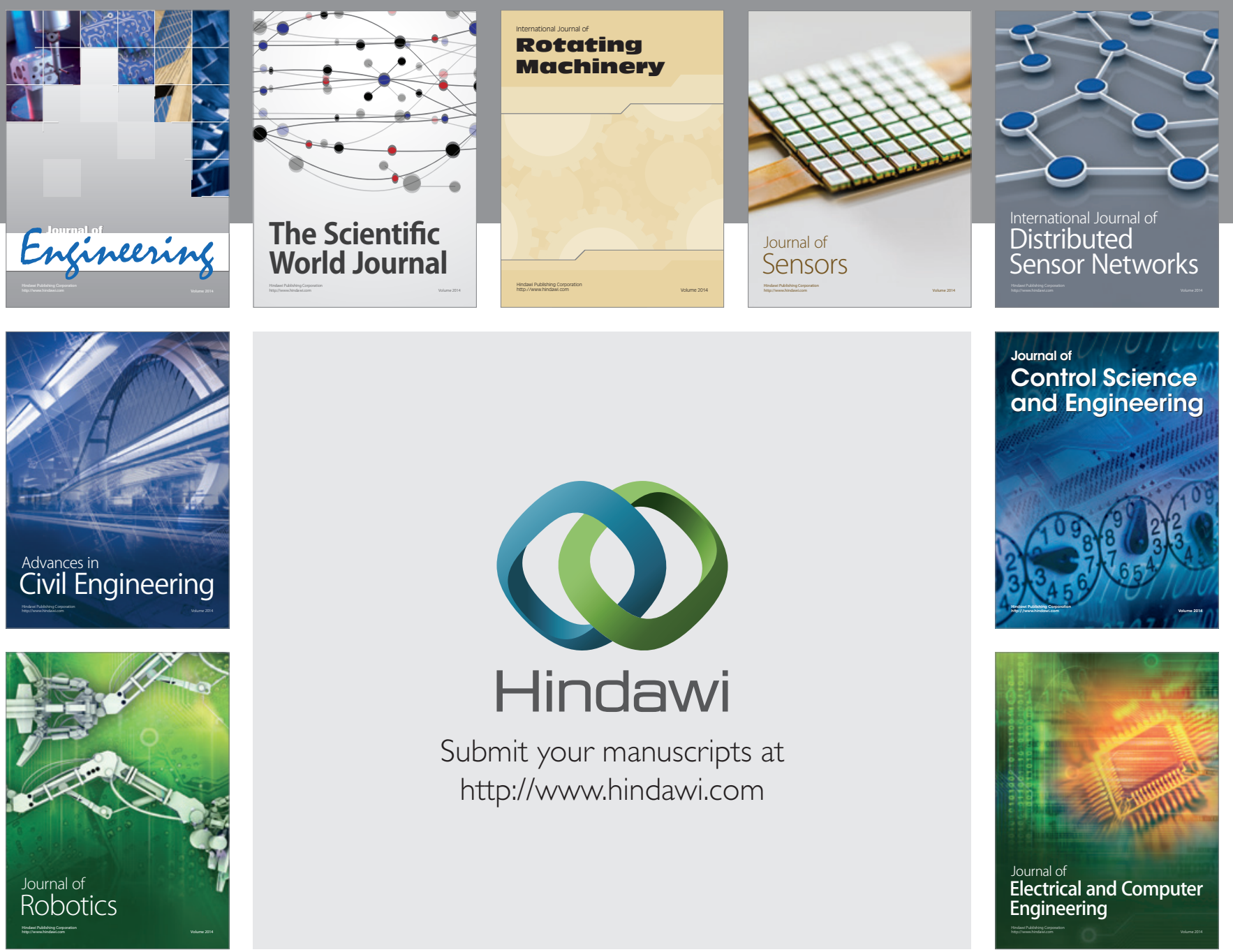

Submit your manuscripts at

http://www.hindawi.com
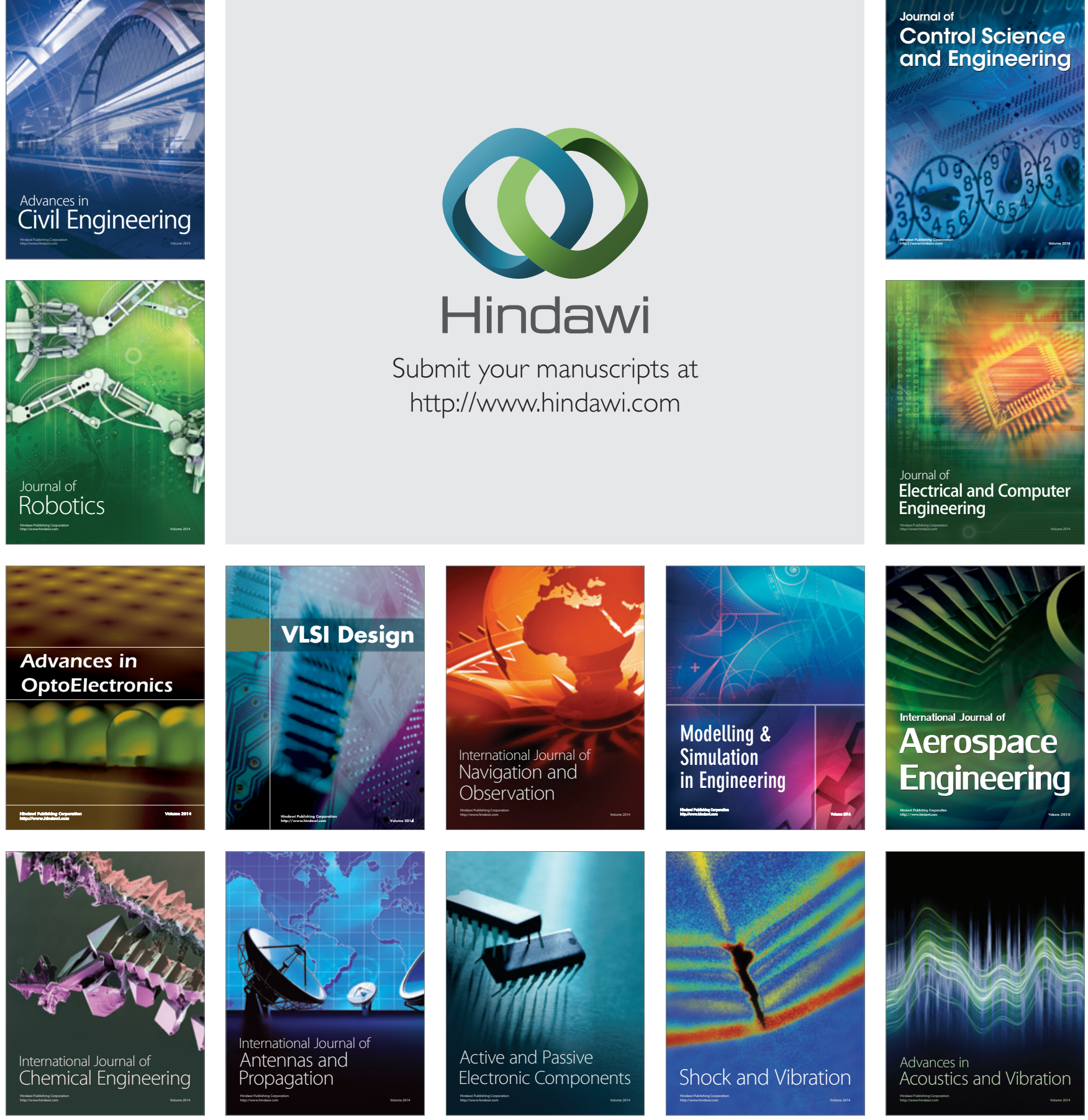